\title{
Influence of the meso-structure in dynamic fracture simulation of concrete under tensile loading
}

\author{
L. Snozzi a , A. Caballero ${ }^{\text {b }}$, J.F. Molinari ${ }^{\text {a,* }}$ \\ a LSMS, ENAC, Swiss Federal Institute of Technology (EPFL), CH-1015, Lausanne, Switzerland \\ ${ }^{\mathrm{b}}$ BBR VT International Ltd, Zürich, Switzerland
}

\section{A R T I C L E I N F O}

\section{Article history:}

Received 8 September 2010

Accepted 30 June 2011

\section{Keywords:}

C. Finite element analysis

C. Tensile properties

C. Micromechanics

E. Concrete

\begin{abstract}
A B S T R A C T
We investigate the dynamic behavior of concrete in relation to its composition within a computational framework (FEM). Concrete is modeled using a meso-mechanical approach in which aggregates and mortar are represented explicitly. Both continuum phases are considered to behave elastically, while nucleation, coalescence and propagation of cracks are modeled using the cohesive-element approach.

In order to understand the loading-rate sensitivity of concrete, we simulate direct tensile-tests for strain rates ranging 1-1000 s ${ }^{-1}$. We investigate the influence of aggregate properties (internal ordering, size distribution and toughness) on peak strength and dissipated fracture energy. We show that a rate independent constitutive law captures the general increase of peak strength with strain rate. However, a phenomenological rate-dependent cohesive law is needed to obtain a better agreement with experiments. Furthermore, at low rates, peak strength is sensitive to the inclusions' toughness, while the matrix dominates the mechanical behavior at high rates.
\end{abstract}

(c) 2011 Elsevier Ltd. All rights reserved.

\section{Introduction}

Structures made of concrete, a highly heterogeneous material, are often subjected to dynamic loads. These may include impact, explosions and seismic loads. Although it is well known that the mechanical behavior of concrete depends on strain rate, the rate dependency of this material under dynamic loading conditions is usually not taken into account in the design codes.

Among others, peak strength, strain at peak strength and total dissipated fracture energy have been shown to be dependent on strain rate, $[22,30]$. In general, concrete exhibits higher peak strength when it is loaded at higher strain rates and this increase has been shown to grow linearly with the strain rate. Fortunately, the augmentation of strength is accompanied by a larger dissipation of fracture energy, so concrete does not become more brittle.

Nevertheless, the dynamic behavior of concrete under high-strainrate tensile loading has still not completely been understood. For instance it is not yet clear what the physical origins of this stress increase are. In addition, the influence of the internal micro structure of concrete, and the mechanisms which lead to different crack patterns when varying the loading rate, remain open questions. Unfortunately performing a proper dynamic experimental test in direct tension brings some difficulties. One of the biggest limitations is how to attach properly the specimen to the testing machine. Therefore, some authors have

\footnotetext{
* Corresponding author. Tel.: + 41 216932411; fax: + 41216936340. E-mail address: jean-francois.molinari@epfl.ch (J.F. Molinari).
}

used, for high strain rates, an indirect method employing the Hopkinson bar [33,3]. In this case, a compressive wave, sent to the specimen, travels through the sample until it finds the free boundary and reflects back already as a tensile wave. Then, the specimen breaks by spalling. However, it is still not clear whether or not the incident compressive wave could have affected the material before the tensile wave initiates its way back.

Concrete is a heterogeneous material made of cement (8-15\%), water $(2-5 \%)$, and about $80 \%$ of aggregates including gravel, sand, limestone filler and finally other chemical additives in proportions smaller than $0.5 \%$. The result of this mixture is a quasi-brittle material in which, the wide kaleidoscope of sizes of the ingredients defines the mechanical behavior. Although all the ingredients influence the final mechanical behavior, it seems to be that every ingredient has an influence according to its characteristic length. In this sense, and according to the levels of observation proposed by Wittman [42], mesoscale $\left(\mathrm{m}^{-3}\right)$ and micro-scale $\left(\mathrm{m}^{-6}\right)$ should be the logical working scales for material scientists in concrete research. Nevertheless, micro-scale is still far from the computational capabilities if 'real' size specimens want to be analyzed. Thus, different meso-scale models have been tested by several authors for the simulation of concrete behavior and fracture. A first class of models has recourse to the finite-element approach, in which concrete is represented either as a mixture of aggregates in a matrix with an interfacial transition zone between them or using a damage formulation $[29,41,6,7,39,24,19]$. A second class to simulate concrete behavior involves lattice models [35,2], where the mechanical properties of the lattice beams aim to represent the concrete mesostructure $[17,12]$. These models have demonstrated success at capturing 
the main characteristics of the overall mechanical behavior of concrete: crack initiation, propagation, coalescence and localization. Moreover the constitutive equations might be relaxed when comparing with a macroscale formulation, thereby reducing the number of model parameters.

This paper aims at exploiting the capabilities of a $2 \mathrm{D}$ (finite-element) meso-mechanical model of concrete. The dynamic propagating cracks will be modeled explicitly by having recourse to the relatively-little used (in concrete modeling) cohesive-element approach. The objectives of our work are twofold. First, we wish to make explicit the influence of the toughness of aggregates on the macroscopic response. To this end, we consider two different types of aggregates differing in their properties (called hard and soft aggregates). The spatial ordering of the mesostructure and the aggregates size are investigated as well. Second, we study the rate dependence of concrete in relation with its mesostructure composition. We propose to adopt a rate dependent cohesive law to reproduce with better accuracy the experimental observations of strain-rate strengthening. While rate dependency has been taken into account in several concrete models, using either a rate-dependent damage formulation [16,9], visco-plasticity [37] or a coupling between both formulations [10], little attempts have been made to couple rate dependency with a meso-scale modeling of concrete. A noteworthy exception includes some recent work, for instance Cusatis et al. [45] within the lattice framework and [26] within a (viscoelastic viscoplastic) damage formulation with interfacial transition zone. Our work will extend this line of thought to the finite-element/cohesive-element approach.

The paper is decomposed as follow. The next section describes the finite-element model and material parameters. Section 3 constitutes the main part of the paper and gathers a mesh convergence analysis and parametric studies showing the influence of aggregates properties, spatial ordering, sizes, and strain rates. A discussion on the advantage of a rate-dependent cohesive law formulation is brought in Section 4.

\section{Approach}

\subsection{Meso-structure generation}

Our model considers concrete as a material made only of two components. Beyond the matrix phase, only the large aggregates are represented explicitly. The small aggregates and other components are assumed to be mixed up with the mortar phase establishing the matrix phase. For the mesh generation the large aggregates are initially represented by means of Voronoï polyhedrons. To generate them, a program starting from a Voronoï mesher has been developed.

To prevent the final Voronoï geometry of presenting tiny edges [48] a Body Centered Cube (BCC) was chosen for the initial distribution of points. The generation points code is linked to the Voronoï generator program and the resulting geometry (in terms of Delaunay mesh) is linked to another code which detects each polyhedron, shrinks, moves and rotates it following an uniform random distribution. As a result, a set of Voronoï polyhedrons surrounded by a box is obtained, Fig. 1.

The matrix phase is created automatically using the capabilities of a mesh generator program by considering the specimen surface (external box) and excluding the Voronoï polyhedrons (aggregates). Notice that this is possible since the cohesive element methodology here does not require an a priori definition of the possible fracture planes, since cracks are constrained to propagate following the interelement boundaries $[29,36,41,7]$.

With this method, we generated 2D (plane strain) square specimens with an external size $h$ of $0.1 \times 0.1 \mathrm{~m}^{2}$.

\subsection{Numerical dynamic approach}

Our so generated finite element meshes are composed of 6-noded quadratic triangles. In order to find the equilibrium, we need to solve the discretized equation of motion

$M \ddot{\vec{x}}+\vec{R}^{\text {int }}=\vec{R}^{\text {ext }}$

To solve this equation one needs to use a convenient finite difference expression to approximate the accelerations and velocities in terms of displacements. We have chosen the explicit version of the popular second order Newmark $\beta$-method. In this case the mass matrix is lumped and therefore diagonal, which allows to trivially inverse the mass matrix solving explicitly the scheme.

The presented scheme is stable under the condition that the time step is below a critical value, which is

$t_{c r i t}=\underset{e=1}{\alpha \min _{e}}\left(\frac{l_{e}}{c}\right)$

where $c$ represents the plain strain stress wave speed and $l_{e}$ is the dimension of the element. The stable time step has to be chosen equal to the smallest value over all elements multiplied with a security coefficient $\alpha$ (typically around 0.1 ).

\subsection{Cohesive zone approach}

The finite-element method with cohesive capability allows us to model dynamic crack propagation and damage in a brittle material like concrete. The fracture process is described by the cohesive approach (introduced by Dugdale [46] and Barenblatt [47]) as a separation process occurring at the crack tip in a small region of material called cohesive zone. In this region, the two faces of the crack separate producing a displacement jump $\vec{\Delta}=\left(\Delta_{n}, \Delta_{s}\right)$. The debonding process is described by a constitutive relation between traction and opening displacement. The bulk material outside the cohesive zone remains undamaged and it continues to behave linear elastically.

\subsubsection{Cohesive zone insertion}

During the simulation, the stress on the interface between two adjacent continuum elements, Fig. 2, is computed and checked against the selected fracture criterion at the end of every time step.

The interfacial stress, $\boldsymbol{\sigma}$, is computed averaging stresses of the adjacent Gauss points of the two continuum elements. This interpolation



Distribution of points



Voronoi Polyhedra



Shrinked Voronoi = aggregates



Matrix

Fig. 1. Schematics of the generation of concrete's meso-structure 

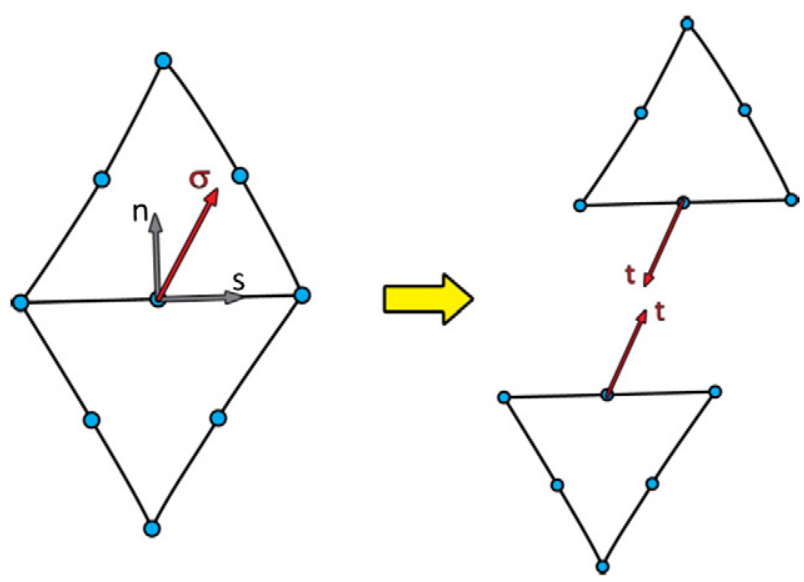

Fig. 2. Schematics of the nodal disconnection between two continuum elements in which the interfacial stress has exceeded the critical stress.

of the interfacial stress, although introduces some small error, converges to the solution as the finite elements become small.

If the inter-element stress exceeds the critical stress value, the middle node located at the inter-element boundary is doubled, the two elements are topologically disconnected and a cohesive element is inserted.

Therefore a crack can propagate only when a cohesive element is inserted adjacent an already existent one allowing the shared corner node to be duplicated as well. After the nodal disconnection, the interfacial stress starts being controlled by the traction separation law, which is implemented in the cohesive element.

\subsubsection{Cohesive constitutive model}

The coupling of the above mentioned fracture criterion together with an initially rigid cohesive law allows capturing the initiation of new cracks without an a priori definition of the possible cracks, which are constrained to propagate following the inter-element boundaries. The law we use is the linear irreversible softening law proposed by Camacho and Ortiz [8,27].

The authors hypothesize the existence of a free potential energy $\phi$, which depends not directly on the relative displacements, $\vec{\Delta}=\left(\Delta_{n}, \Delta_{s}\right)$, but in an effective scalar displacement which has the following form:

$\delta=\sqrt{\Delta_{n}^{2}+\beta^{2} \Delta_{s}^{2}}$.

The parameter $\beta$ accounts for the coupling between normal and tangential displacement. The value of $\beta$ has to be estimated (e.g. by correlating experimental results with numerical simulations, [34]), and is therefore bound with some uncertainty. We investigated the influence of that parameter by varying its value between zero (only normal tractions) and 10 . We could establish that the peak strength is only slightly affected by $\beta$, as we are applying a tensile loading. For the remainder of the paper we will keep its value equal to one. The free energy function has the form in the reversible case

$\phi(\delta)=\frac{1}{2} f_{c t} \delta\left(2-\frac{\delta}{\delta_{c}}\right)$

where $f_{c t}$ represents the local material strength and $\delta_{c}$ represents the effective relative displacement beyond which complete decohesion occurs. The derivation of the free potential energy with respect to the opening displacement leads to the cohesive tractions:

$\vec{t}=\frac{\partial \phi}{\partial \vec{\Delta}}=\frac{t}{\delta}\left(\Delta_{n} \vec{n}+\beta^{2} \vec{\Delta}_{s}\right)$ where $t$ represents an effective cohesive traction. This traction in case of crack opening is given by:

$t=f_{c t}\left(1-\frac{\delta}{\delta_{c}}\right) \quad$ for $\delta=\delta_{\max }$ and $\dot{\delta}>0$

Whereas for crack closure or reopening ( $\delta$ smaller than $\delta_{\max }$ ) the functional form is assumed to have the form:

$t=t_{\max } \frac{\delta}{\delta_{\max }} \quad$ for $\delta<\delta_{\max }$

where $t_{\max }$ is the value of the effective traction when $\delta$ is equal to $\delta_{\max }$, in which the maximal effective opening displacement attained up to the moment is stored. Moreover $\delta_{\max }$ also accounts for the irreversibility of the law allowing successive loading, unloading and reloading. The evolution of the linear decreasing law is graphically shown in Fig. 3.

Note that the definition of $f_{c t}$ and $\delta_{c}$ implicitly establishes the existence of an effective fracture energy $G_{c}$, which corresponds to the area under the curve of Fig. 3:

$G_{c}=\frac{1}{2} f_{c t} \delta_{c}$

Partially damaged cohesive elements have dissipated an energy $W$.

\subsection{Material parameters}

Meso-mechanical models need to be fed with specific material parameters, which may require specific experiments. In particular, fracture properties of the components (aggregate, matrix, which includes mortar and small aggregates, and aggregate-matrix interface) are needed.

In real concrete, the value of those parameters influences strongly the macroscopic mechanical behavior. In particular, the strength and stiffness of large aggregates (with respect to the matrix's ones) and the strength of the aggregate-matrix interface are directed correlated with the final experimental results, i.e. cracking patterns, fracture energy, critical crack opening, softening curve and Young's modulus [11].

This work only considers mean values of those parameters. Nevertheless two different types of aggregates have been considered. In Table 1, a summary of the chosen material parameters is given.

In order to validate our model, we have performed two simulations using the same specimen geometry of the quasi-static tests presented in one of the references [31] from which the aggregate and matrix properties have been taken. The numerical results lie within the experimental scatter and were therefore in good agreement with the experimental load displacement behavior.



Fig. 3. Graphical representation of the linear cohesive law. 
Table 1

Summary of fracture material properties taken from [32,31].

\begin{tabular}{llllll}
\hline Material properties & Density $\left(\mathrm{kg} / \mathrm{m}^{3}\right)$ & $\mathrm{E}(\mathrm{GPa})$ & $v$ & $\mathrm{G}_{\mathrm{c}}\left(\mathrm{J} / \mathrm{m}^{2}\right)$ & $\mathrm{f}_{\mathrm{ct}}(\mathrm{MPa})$ \\
\hline Hard aggregate & $2700^{\mathrm{a}}$ & 19 & 0.2 & 60 & 16 \\
Soft aggregate & $2700^{\mathrm{a}}$ & 2.1 & 0.2 & 20 & 1.7 \\
Matrix & $2200^{\mathrm{a}}$ & 31 & 0.2 & 52 & 4.7 \\
Aggretate-matrix & - & - & - & 30 & $4.7^{\mathrm{b}}$
\end{tabular}

a These values are generic and chosen from Caballero [48].

b This is an estimated value, set equal to the matrix-matrix interface strength, as direct experimental data was not found.

\subsection{Measure of damage}

When using continuum damage models, damage is defined as a variable at the constitutive level. The visualization of this variable can therefore give an approximate idea about the irreversible deformation of a structure and the underlying damage. On the contrary, with the cohesive element approach, cracks are modeled explicitly, giving an opportunity to construct relevant damage indicators and to monitor their time evolution. To this effect, we defined the following variable.

\subsubsection{Damage normalized fracture work (DNFW)}

The index accounts for the ratio between the work fracture spent (already dissipated fracture energy $W$ ) and the fracture energy necessary to split the interior specimen in two, i.e. it is the fracture energy corresponding to a length equal to $h$, which is the specimen width (this allows to have an index insensitive to the mesh size).

$D N F W=\frac{\sum_{i=1}^{\text {nbinterfaces }} \int_{l i} W_{i} d l_{i}}{\bar{G}_{c} \cdot h}$

Where $l_{i}$ is the length of cohesive element $I, W_{i}$ the partially dissipated cohesive energy and $\bar{G}_{c}$ represents a mean fracture energy, weighted with respect to the amount of fracture energy potentially released by each inter-element component.

In order to distinguish and to localize damage, the above damage variable has been also defined for each material phase: aggregates, matrix and aggregate matrix, and can be indentified (as for the other variables) with a corresponding subscript: $a, a-m$ and $m$.

\section{Results}

\subsection{Geometry and boundary conditions}

All the specimens are loaded under displacement control. To avoid stress wave propagation from the boundaries and early fracture near the boundaries, all the nodes computations are given an initial velocity, [23], see Fig. 4.


Fig. 4. Boundary (left) and initial (right) conditions.
Nodes of the finite element mesh which are located on the upper and lower boundaries are forced to move at a certain constant velocity $v_{0}$ :

$v_{0}=\dot{\varepsilon} \frac{h}{2}$

where $\varepsilon$ is the strain rate and $h$ the height of the specimen. The upper boundary respectively the lower one moves upward respectively downward at velocity $v_{0}$. To avoid stress wave propagation from the boundaries and early fracture near the boundaries, all the nodes computations are given an initial velocity in respect to their vertical position $y$ [23] as illustrated in Fig. 4.

$v_{y}(y)=\frac{2 v_{0}}{h} y$

In the following subsections the work is focused first on the convergence of the simulations (Section 3.2); later an investigation on the influence of the aggregates toughness (Section 3.3) and their ordering (Section 3.4) and size (Section 3.5) is given.

\subsection{Effect of mesh size, geometry and material parameters}

The fracture process in brittle solids is usually assumed to be a toughness controlled phenomenon, which means that the process is controlled by $G_{c}$ and is therefore independent of $f_{c t}$ of the cohesive zone.

Conversely, if the macroscopic strength is linked with $f_{c t}$, the process is said to be strength controlled. This must be valid not only theoretically but also when running a numerical simulation, which uses the cohesive zone approach.

It is well known that the relationship between the cohesive law and the critical energy release rate $G_{c}$ introduces a length scale into the material description. This imposes a constraint on the mesh size. This length scale, calculated by Rice and Palmer [28,25], is called cohesive zone length $l_{z}$ and is defined as follows for Mode I crack loading in case of plane strain

$l_{z}=\frac{9}{32} \frac{\pi E G_{c}}{\left(1-v^{2}\right) f_{c t}^{2}}$.

This length has an important influence around the crack tip in numerical simulation. In general, for toughness-controlled fracture, the following relation has to be satisfied:

$l_{m}<l_{z}<h$

where $l_{m}$ is the element size (biggest element edge) in the cohesive area and $h$ is the width of the specimen $(0.1 \mathrm{~m})$. This means that the cohesive zone must contains several elements (typically around four) and that the cohesive zone should be small in comparison to the specimen size. Table 2 shows the calculated values of the cohesive zone length for the different components of concrete.

Note that it is not possible to clearly calculate a cohesive zone length for the interfaces between aggregates and matrix (because the choice of Young's modulus is ambiguous). The cohesive zone length for the hard aggregates and that one between soft aggregate and

Table 2

Cohesive zone lengths $\mathrm{l}_{\mathrm{z}}[\mathrm{mm}]$ for the different components of concrete meso-scale model. For Aggregate-Matrix interfaces: $E_{M}$ means that Matrix Young's Modulus is considered, $\mathrm{E}_{\mathrm{HA}}$ and $\mathrm{E}_{\mathrm{SA}}$ this of hard respectively soft aggregates.

\begin{tabular}{|c|c|c|c|c|c|c|}
\hline \multirow[t]{2}{*}{ Material } & \multirow{2}{*}{$\begin{array}{l}\text { Hard } \\
\text { Aggregate }\end{array}$} & \multirow{2}{*}{$\begin{array}{l}\text { Soft } \\
\text { Aggregate }\end{array}$} & \multirow[t]{2}{*}{ Matrix } & \multicolumn{3}{|c|}{ Aggregate-matrix } \\
\hline & & & & $\overline{E_{\mathrm{M}}}$ & $E_{\mathrm{HA}}$ & $\overline{E_{\mathrm{SA}}}$ \\
\hline Cohesive zone length [mm] & 4.1 & 13.4 & 67.2 & 38.8 & 23.7 & 2.6 \\
\hline
\end{tabular}





Fig. 5. Macroscopic peak strength $\sigma_{\max }[\mathrm{MPa}]$ versus average element size $\mathrm{l}_{\mathrm{m}}[\mathrm{mm}]$ for a) $\varepsilon=10 \mathrm{~s}^{-1}$ and b) $\varepsilon=1000 \mathrm{~s}^{-1}$ (SA).

matrix have the smallest values; therefore they will determine an upper limit for the size of the elements when generating the mesh.

In order to verify the relationship between the different length scales a series of numerical tests was carried out. A first test was simply to load the $0.1 \times 0.1 \mathrm{~m}$ concrete specimen varying the degree of refinement of the mesh in order to verify the mesh sensitivity. We ran several simulations for concrete with soft and hard inclusions for different loading rates. The results for two different loading rates and concrete with soft aggregates are reported in Fig. 5.

From Fig. 5a) it is possible to observe that there is convergence for an average element length $\left(l_{\mathrm{m}}\right)$ smaller than about $0.25 \mathrm{~mm}$. Considering this length and looking back at Table 2 we can remark that $l_{m}$ should be at least roughly ten times smaller than the Rice's cohesive zone length of the interface, which was derived for precracked specimen with homogeneous materials and without mesostructure. Most published work having recourse to the cohesiveelement framework uses an estimate of $l_{m} \leq l_{z} / 3$. Our work demonstrates the importance of using a more conservative estimate in the absence of a complete mesh-convergence study. The scenario is similar at high strain rates (e.g. beyond $100 \mathrm{~s}^{-1}$ ), Fig. 5b), although the peak strength is higher and apparently a finer mesh is needed to reach a fully converged results. The chosen mesh-size for the simulations presented in this paper is set to $0.4 \mathrm{~mm}$. Although this value seems to be related with some limited mesh dependency, it allowed us to save some computational time.

A closer inspection of Table 2 reveals yet another challenge. The matrix cohesive length has roughly the same dimension of the tested specimen; therefore the relation of Eq. (12) is not respected. We carried out a second study in order to highlight the influence of this second requirement on the macroscopic strength. A pre-cracked specimen (Fig. 6a)), made only of one component, has been chosen for this test. The specimen is loaded with a strain rate of $10 \mathrm{~s}^{-1}$, the horizontal displacements of the left-boundary nodes were blocked and the matrix-component was selected as reference material. We ran different calculations keeping $\mathrm{G}_{\mathrm{c}}$ constant $\left(52 \mathrm{~J} / \mathrm{m}^{2}\right)$ and varying the critical opening strength $f_{\mathrm{ct}}$ between $1.175 \mathrm{Mpa}\left(0.25 \cdot f_{\mathrm{ct}, \mathrm{m}}\right)$ and $75.2 \mathrm{Mpa}\left(16 \cdot f_{\mathrm{ct}, \mathrm{m}}\right)$. According to the previous investigation of convergence, the mesh has been refined in the fracture area, where the elements have an average edge length of $0.5 \mathrm{~mm}$.

The results are displayed in Fig. 6b). One can distinguish three domains. On the first one as on the third one, the macroscopic strength is clearly an increasing function of $f_{c t}$, while region two is staying quite constant. The domain two is clearly the toughness-controlled one, whereas domain one and three are strength-controlled.

On domain one, $l_{z}$ is bigger than $0.01 \mathrm{~m}$, which is a too big value compared to the specimen size $h(0.1 \mathrm{~m})$. On the third domain the a



b




cohesive length as for soft respectively hard aggregates. 




Fig. 7. Pre-cracked specimen with variable width.

cohesive zone becomes too small compared to the size of the chosen mesh $\left(l_{z}<2.5 \mathrm{~mm}\right)$, which means that the mesh is not fine enough to capture the cohesive zone. The cohesive lengths for matrix (round symbol) and soft aggregates (square symbol) are included in domain one. Whereas, for hard aggregates (triangular symbol), the cohesive length is located in the toughness-controlled region (domain two).

We further carried two tests to quantify the influence of the chosen specimen size on the peak strength. Both tests are conducted for a fixed mesh size. One consists in taking a specimen with a regular meso-structure and the standard geometry tested in this paper $(0.1 \times 0.1 \mathrm{~m})$, but with a pre-crack. The specimen width has been increased by keeping the height constant (Fig. 7).

We could observe (Fig. 8a)) that the peak strength decreases with increasing $h$ and converges to a fixed value for a width of approximately $0.6 \mathrm{~m}$ (which corresponds roughly to ten times the cohesive zone length for the matrix).

The other test consists in enlarging the size of the specimen keeping the same degree of mesh refinement (Fig. 8b)). We have run simulations with double and quadruple specimen size. In this case the size does not seem to affect the peak strength (only a small variation in order of two percent could be observed). When comparing the results of the two specimens, one can remark that, if there is a precrack, the size of the specimen clearly affects the peak strength as the cohesive length of the matrix is in the same order as the chosen specimen width. Whereas, if the sample is not pre-cracked, it seems that the chosen size has not the same influence (as long as the chosen specimen size can be considered a representative volume element).

For the chosen specimen geometry, and material parameters, the presented results are not in a well-defined region. We have tried to reduce as much as possible the mesh dependency, but this is complicated by the presence of a meso-structure. The concept of toughness-controlled regime applies well for homogeneous materials and pre-cracked specimen. However, in our case, where multiple cracks are opening (during the simulations) and interacting, the upper limit for the specimen size of Eq. (13) has to be relaxed.



Fig. 9. Concrete meso-geometry with 79 aggregates (left). Zoom of the corresponding finite-element mesh (right).

Moreover, because of the meso-structure, which is composed of different materials, is not possible to clearly define a cohesive zone length. Without a rigorous estimate, it was decided to use a mesh size smaller than a sixth than the smallest cohesive zone estimate.

Thus, it can be concluded that, for the geometry of the concrete specimens presented in this paper, the present work lies in a mixed strength-controlled and toughness-controlled regime.

\subsection{Soft vs. Hard aggregates and strain rate effect}

To study the influence of the mechanical properties of the aggregates on the behavior of concrete, two different types of aggregates, differing in their material properties, have been considered: soft and hard (see Table 1). The concrete meso-structure is modeled with the geometry and mesh presented in Fig. 9.

The response of the two types of concrete depends on the strain rate at which the specimen is loaded. For instance, two stress-strain diagrams are plotted in Fig. 10a) for a low strain rate $\left(\dot{\varepsilon}=10 s^{-1}\right)$, b) for a high strain rate $\left(\dot{\varepsilon}=1000 s^{-1}\right)$.

From Fig. 10a) one can recognize that the aggregate type influences the response of the concrete specimen only at low strain rates. As mentioned previously, we keep the value of $\beta$, the coupling parameter in the cohesive law, equal to 1 . Since we are in mode I, this parameter does not influence significantly the peak strength (a study of the influence of $\beta$ was conducted but is not shown here). The variation of peak strength with strain rate for the two different aggregates is highlighted in Fig. 11a). The results display a strain rate hardening. However, the computed increase of strength is quite smaller than the experimental one see Fig. 11b). We will go back to $\mathrm{a}$



b



Fig. 8. a) Macroscopic normalized peak strength $\sigma_{\max }$ versus specimen with $\mathrm{h}[\mathrm{m}]$. b) Specimens with double and quadruple size. 
a



b



Fig. 10. Macroscopic stress-strain $(\sigma-\varepsilon)$ comparison between hard and soft aggregates: a) at low strain rate $\left(\dot{\varepsilon}=10 \mathrm{~s}^{-1}\right)$ and $\left.\mathrm{b}\right)$ at high strain rate $\left(\dot{\varepsilon}=1000 \mathrm{~s}^{-1}\right)$.

this point in Section 4, where we motivate the use of a phenomenological rate dependent cohesive law.

Under a low strain rate, the presence of soft aggregates within concrete makes the material softer in its pre-peak behavior. However, this effect starts to disappear when the strain rate is increased above $10 \mathrm{~s}^{-1}$. The peak strength is also decreased by the presence of soft aggregates when concrete is loaded under low strain rates (the peak strength for hard aggregates is approximately double than for the soft ones). Finally, at low $\dot{\varepsilon}$ the softening branch shows a more attenuated decay when comparing soft vs. hard aggregates.

This scenario changes completely when analyzing the same specimens under high strain rate, Fig. 10b). The results show that there are no differences between soft and hard aggregates in terms of stress-strain diagrams. The stress-strain plots are, almost identical, exhibiting same elastic slope, peak strength and softening decay up to the last computed time.

The same distinction between the behavior at low and high strain rates can be done when looking at the deformed mesh configuration, which is plotted in Fig. 12 for the two different types of aggregates.

One can remark in the case of hard aggregates, that at low strain rates some macro-cracks propagate in the matrix avoiding the aggregates, whereas the cracks cross the aggregates if soft inclusions are chosen. Therefore the failure process is dominated by crack propagation. By increasing the loading rate cracks start to nucleate in the matrix and have less time to propagate before the peak strength is reached. At high strain rate $\left(1000 \mathrm{~s}^{-1}\right)$ the crack path for soft and hard aggregates is the same. This means that the peak strength is dominated by crack initiation.

This phenomenon can also be highlighted when considering the damage normalized fracture work.

Fig. 13 shows the damage diagrams for each of the different concrete phases: aggregates, matrix and aggregate-matrix interfaces respectively. In all figures, damage is plotted against the total tensile strain applied to the specimen, which is measured as the total elongation divided by the original length of the specimen. Soft aggregates (SA) are compared vs. hard aggregates (HA) under both: a) low and b) high strain rates.

As noticed from the deformed mesh configuration, under low strain rate hard aggregates do not experience any damage, whereas the damage on soft aggregates increases with increasing strain level. For both aggregates the damage in the matrix appears first. This is congruent with the fact that at early stages of loading, i.e. before peak strength, a large amount of micro cracks starts to be nucleated within the matrix. With increasing strain those micro-cracks begin to propagate and therefore damage outside from the matrix phase can begin.



following experimental data: $[5,30,1,38,21,15]$ (note that the vertical axis is normalized by the static strength). 
a



b



$\mathrm{c}$



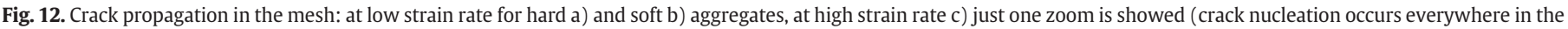
matrix independently of aggregate type).

When a tensile wave traveling throughout the matrix reaches an aggregate, it induces a tensile strain on it. However, the deformation that the aggregate will experience will depend on the Young modulus ratio between the matrix and the aggregate. Thereby, the stiffer is the aggregate with respect to the matrix, the lower the deformation on the aggregate will be. This implies a larger opening of the interface. Therefore, the introduction of aggregates much harder than the surrounding matrix will concentrate the damage on the interfaces rather than in the inclusions as in the case of soft aggregates.

Damage in the matrix for hard aggregates is also higher when compared with soft aggregates. This agrees with a larger value of dissipated fracture energy and higher peak strength. After the peak strength has been reached and the softening behavior starts to be more accentuated the rate of fracture energy dissipation decreases for the damage indicators of the three components.

At high strain rates the damage is localized mainly in the matrix. The high loading rate does not allow cracks to propagate before the peak strength has been reached. Micro cracks start to nucleate everywhere in the matrix, which displays the highest stress wave speed and therefore crack propagation speed too. This more diffuse micro-cracking is related to the intrinsic characteristic opening time of the cohesive law (derived in Camacho et al. [8]). Therefore, for our computations, we cannot explain the increase of strength with a more transgranular fracture [43].

Interesting, the dissipated fracture energy at peak strength seems to be only slightly affected by the loading rate. However, post-peak dissipation of energy rises with increasing strain rate and is clearly an increasing function of the loading velocity.

The presence of two different types of inclusions not only affects the damage value but also the crack pattern and its evolution. To this fact we consider the variable, $\mathrm{N}_{\text {clusters, }}$ which accounts for the number



of "clusters" present in the specimen. A cluster is a group of cracks (i.e. a group of fully broken interfaces for which the opening of the cohesive elements is beyond $\delta_{c}$ ), which are interconnected to each other.

In general there is a relation between strain rate and when the clusters appear. Relatively, a higher strain rate delays the formation of the first clusters. At low strain rates $\left(10 \mathrm{~s}^{-1}\right)$, the first clusters appear for soft aggregates just before the peak strength has been reached, whereas for hard aggregates they appear immediately after (the process being more energy consuming). With increasing strain rate, the clusters will appear only when a bigger amount of fracture energy has been spent and the peak strength will be reached with a more diffuse nucleation of micro-cracks. Therefore, unlike for ceramics where the material breaks down when a cluster has reached a length corresponding approximately to the specimen size [14], it seems that it is not possible to find a critical cluster length to be related with the failure of the material.

\subsection{Orderered vs disordered meso-structure}

This section discusses the effect that the meso-structure has on the final fracturing behavior of concrete. The main target is to evaluate what is the influence of the location of aggregates and their external shape. Three different meso-structures are considered:

- Fully uniform, where all the aggregates have the same shape (excepted those located near to the borders due to the Voronoï generation process), same orientation and are uniformly distributed. This distribution will be referred as U.

- Fully uniform plus random rotation, where as in the previous distribution all the aggregates have same external shape but they



Fig. 13. Damage on aggregates, matrix and interfaces vs. tensile-strain. Comparison between soft and hard aggregates: a) low strain rate, b) high strain rate. 
a

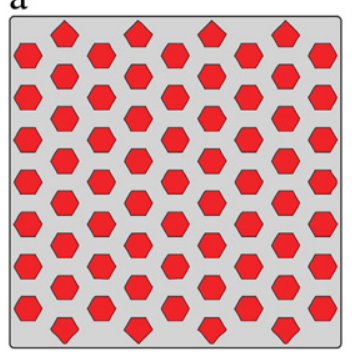

b

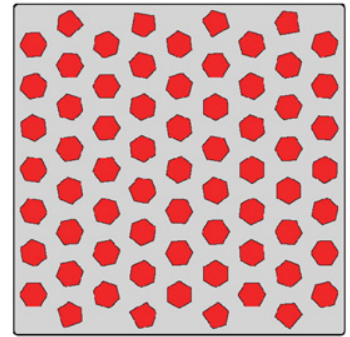

c

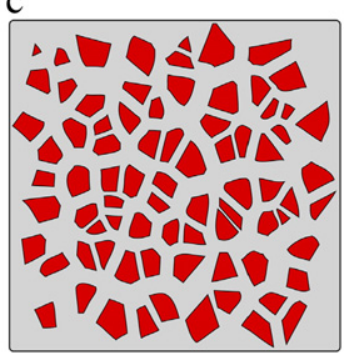

Fig. 14. Different meso-structures: a) uniform aggregates $(U), b)$ rotated aggregates $\left(U_{R o t}\right)$ and c) completely random (R).

are randomly rotated with respect to their own center. This distribution will be referred as $U_{\text {Rot }}$.

- Fully random, aggregate's shape and position are randomly assigned. This distribution will be referred as $\mathrm{R}$.

Fig. 14 shows the three different meso-structures considered. All the meso-structures have the same ratio of aggregate volume, 30\%, although due to the randomness different number of aggregates. Thus, $\mathrm{U}$ and Rot meso-structures have 67 aggregates of approximately $8.5 \mathrm{~mm}$ whereas $\mathrm{R}$ distribution has 79 aggregates, where most aggregates have sizes between 6 and $10 \mathrm{~mm}$. All the computations have been carried out considering particles as hard aggregates, since the influence of the meso-structure is not significant for the soft inclusions (which are weaker than matrix grains interfaces).

Fig. 15 plots the stress-strain diagrams for the different mesostructures under low (a) and high (b) strain rate. From Fig. 15 we can extract the following conclusions.

The initial elastic slope is not affected by the meso-structure.

The peak strength is always lower for the U distribution. This is probably due to the fact that, in the $U$ distribution, all the aggregates have two faces orthogonally aligned to the loading direction, which reduces the maximum strength. Indeed, considering the dissipated fracture energy of the interfaces (Fig. 16) for the U specimen, one can remark that it is larger than those of the other two meso-structures $\left(U_{\text {Rot }}\right.$ and $\left.R\right)$. On the contrary, the dissipated fracture energy of the matrix is smaller for the $U$ specimen.

The preferential direction of failure is avoided in the $U_{\text {Rot }}$ and $R$ distributions. Comparing $U_{\text {Rot }}$ and $R$ specimens, the fully disordered meso-structure increases the possibility of having weak zones (grainmatrix interfaces are closer in some places). In such regions, damage might grow faster and therefore the global mechanical response softens earlier.
Finally, material softening is slightly more attenuated in the $\mathrm{R}$ distribution. As mentioned before, the $\mathrm{R}$ distribution contains "weak zones", which facilitates the initiation of cracks and their local growth. However, after an initial growth and nucleation of cracks, the disordered meso-structure may obstruct the further propagation of cracks (we are under the assumption of hard aggregates), and forces the cracks to deviate around the aggregates, which requires more energy (the dissipated fracture energy in the matrix of the R distribution is slightly higher compared with the one of $U_{R o t}$ distribution).

\subsection{Influence of aggregate's size}

In this section the effect of the aggregate's size is analyzed. Three different specimens with the same standard geometry of $0.10 \times 0.10 \mathrm{~m}^{2}$ are considered. In all specimens, volume ratio of aggregates is kept constant and equal to $30 \%$, whereas the size of the aggregate is decreased as the number of inclusions is increased. The average aggregate size of each specimen is: $8.5 \mathrm{~mm}, 3.9 \mathrm{~mm}$ and $2 \mathrm{~mm}$ (corresponding to a number of aggregates of 66, 297 and 1198). The three different specimens considered are shown in Fig. 17. Both types of aggregates, soft and hard, are considered.

Fig. 18 shows the obtained stress-strain diagrams for the different concrete specimens under low and high strain rates. At both loading rates the elastic modulus appears to be independent of the aggregate size. At low loading speed the peak strength for hard aggregates is not significantly affected by the number of inclusions (not shown in figure). Whereas, for soft aggregates, it is possible to notice an increase in peak strength with increasing number of aggregates (Fig. 18a).

In a concrete material with large aggregates (which represent weak zones in the case of soft aggregates) cracks can nucleate before and propagate easier within the inclusions than in a concrete with



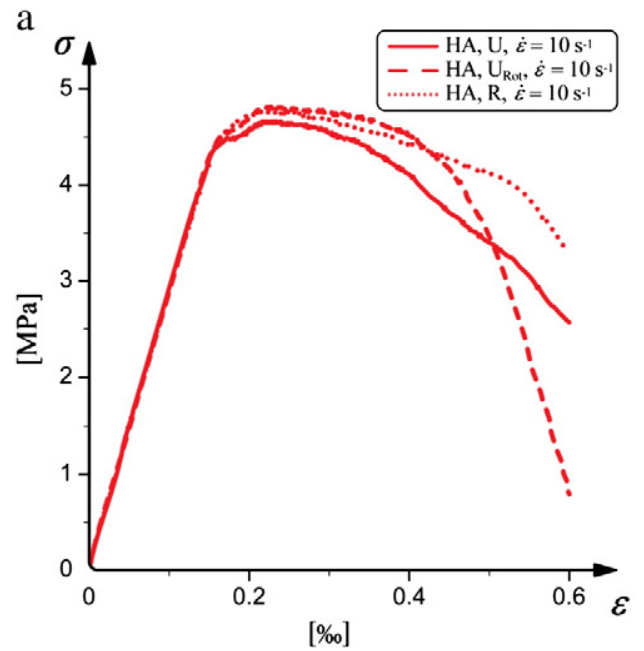

Fig. 15. Stress-strain diagrams obtained for the three different meso-structures under a) low and b) high strain rate. 


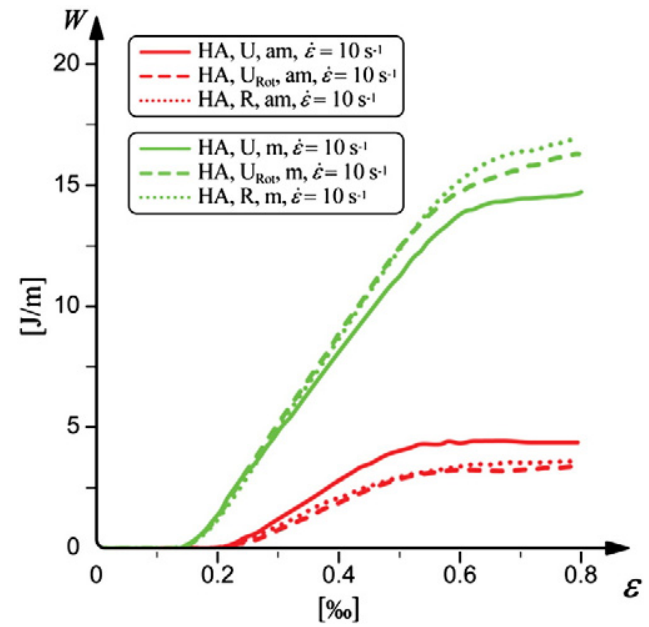

Fig. 16. Dissipated fracture energy for the matrix (in green) and interfaces (in red) vs. tensile strain. Comparison between specimens with the three different mesostructures.

small aggregates. Indeed, cracks nucleate first in the matrix and then propagate from the matrix in the aggregates too. Therefore, if the aggregates are bigger, the cracks will cross them easier breaking a larger area of aggregates leading to lower peak strength.

This phenomenon can also be observed when looking at the graph for the dissipated fracture energy (Fig. 19a)). The dissipated energy in the matrix increases with increasing number of aggregates. In contrast, the aggregate's dissipated energy reduces with decreasing size of inclusions.
The scenario changes when considering high strain rates (Fig. 18b)). In this case the peak strength is higher for large inclusions (independently of aggregate type). We recall that at high strain rate the cracks nucleate in the matrix and aggregate-matrix interfaces leading to a similar stress-strain behavior for the two types of inclusions. Therefore, even though aggregates seem not be directly the cause of the variation of the strength, a larger number of aggregates implies also a higher probability for a growing crack to find an interface between mortar and aggregates (interfaces are weaker than matrix).

As shown in Table 3, for the same volume ratio of inclusions, the total length of the interfaces (between aggregates and mortar) increases roughly four times between the 66 and 1198 aggregates specimens. This is congruent with a decreasing ratio of dissipated fracture energy at the interfaces with increasing aggregates size (Fig. 19b)).

\section{A rate-dependent cohesive law}

In this section we discuss the limit of the proposed approach. Although, the presented meso-mechanical model is bound with a certain freedom, there are also some limitations. Indeed, (as noticed in the results section) when comparing our results with experimental ones, it is possible to remark that the rate-independent cohesive law does not allow to have a strain-rate hardening comparable with the experimental one. Moreover the dissipated fracture energy at peak in the simulations seems to be rougly independent of strain rate (however post peak dissipation of energy is clearly an increasing function of strain rate).

Whereas, experimental results have shown that energy dissipation increases with the loading rate [33,3]. Although, there is a general agreement on this phenomenological behavior, it is still not clear
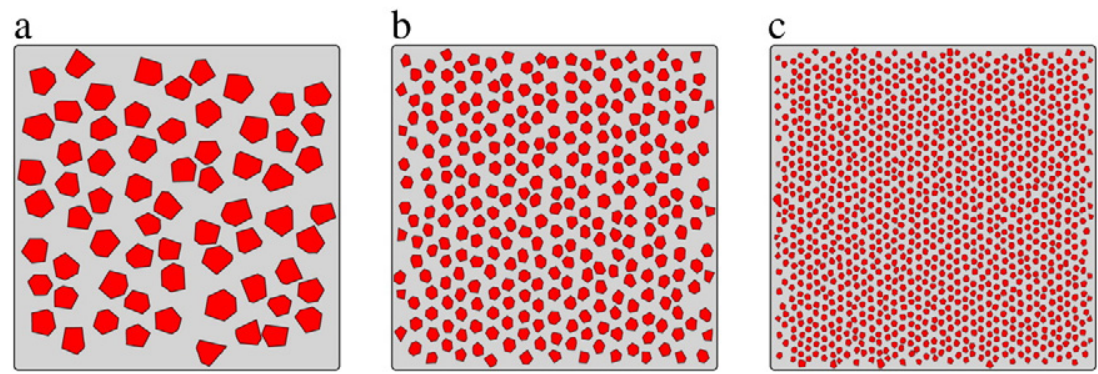

Fig. 17. Specimens with different mean size of aggregates: a) 66 aggregates, b) 297 aggregates and c) 1198 aggregates.
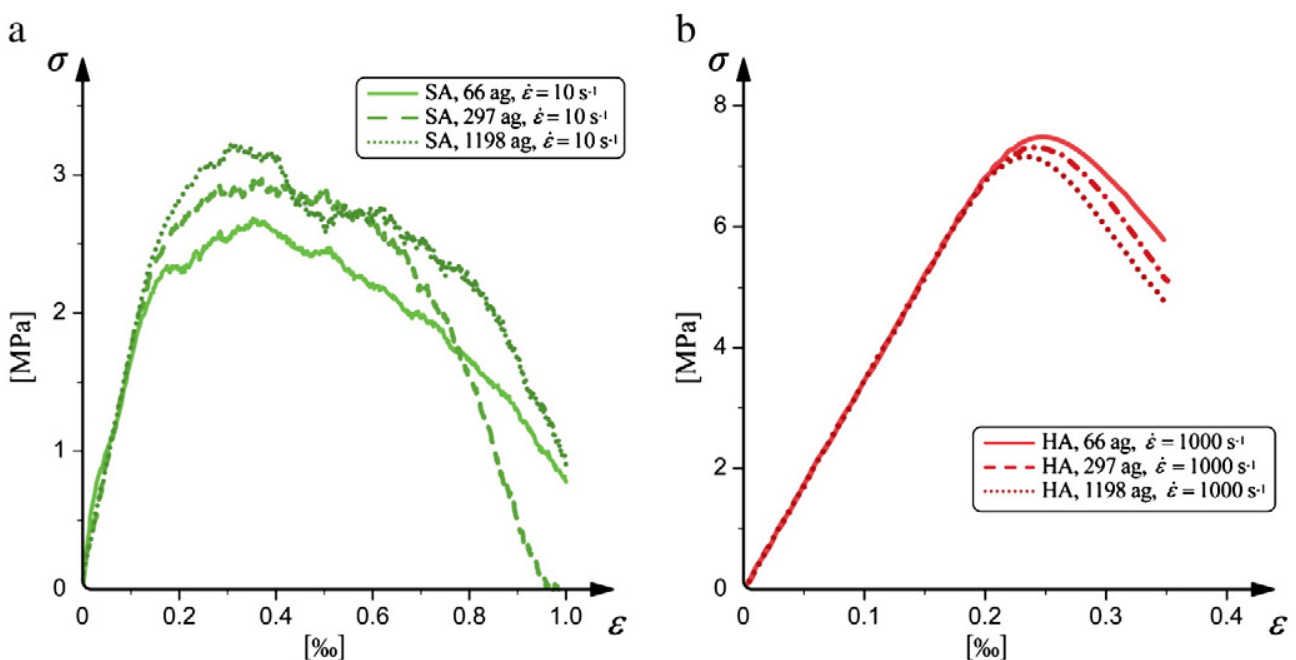

Fig. 18. Stress-strain diagrams obtained for the different aggregate sizes under a) low and b) high strain rate. 

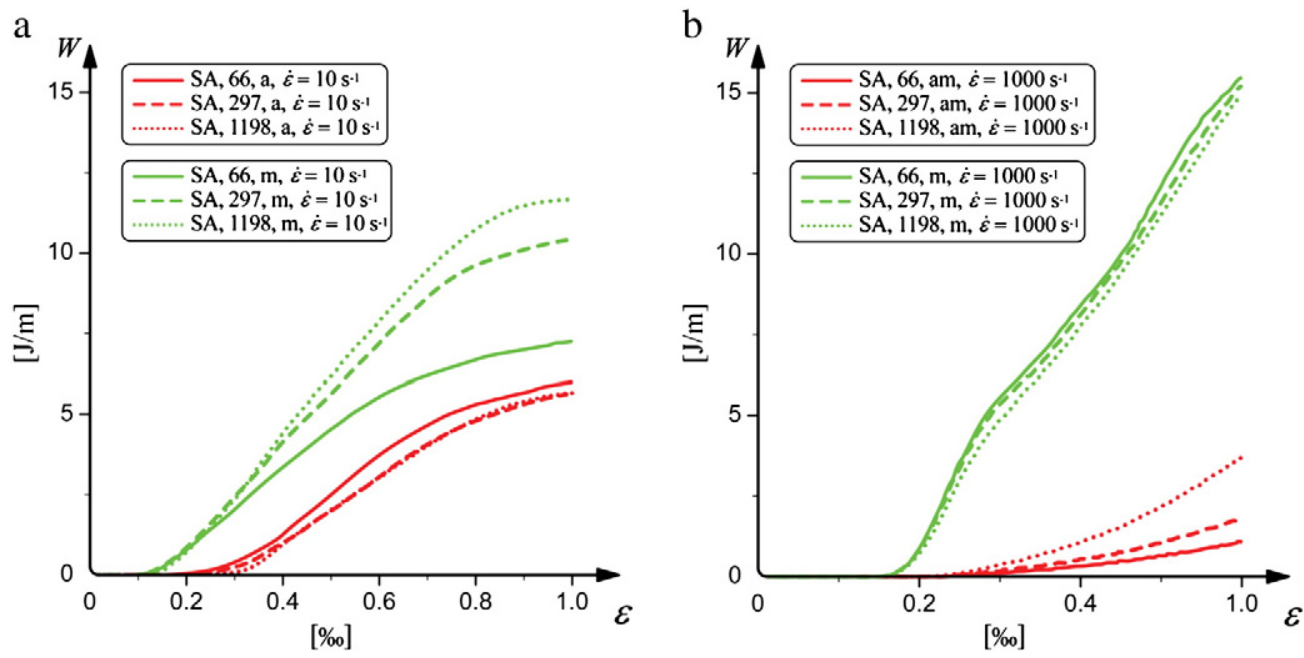

Fig. 19. Dissipated fracture energy vs. tensile-strain. Comparison between specimens with 66, 297 and 1198 aggregate: a) at low strain rate and b) at high strain rate.

which should be the mechanisms or physics which govern it. The increase is attributed to two different aspects by different authors, structural or material. According to the structural effect hypothesis, the explanation resides in a more diffuse micro-cracking, which should be linked with higher fracture energy $[40,3]$. On the contrary, the material explanations sustains that the increase of toughness could be an effect of internal material "hardening" [20]. We argue that this mechanism should be related to the chosen modeling scale. I.e. when considering a large modeling scale (macro-scale), the toughness parameter of the cohesive approach could be a function of the loading rate, as the mesh size cannot be small enough to capture all micro-cracking mechanism. Whereas, decreasing the scale should entail a rate independent toughness parameter.

Nevertheless, the small computed amount of dissipated fracture energy (the dissipated fracture energy at peak strength seems to be roughly independent of strain rate) and the small increase in strength with increasing loading rate in comparison to experiments seems to call for the introduction of a phenomenological rate-dependent cohesive law. Therefore, we decided to slightly modify the lineardecaying cohesive law in order to take into account the strain rate effect. Among the various possible laws we identified two different ones differing in their shape and which are also function of different rate-dependent parameters.

The first variant [44] assumes that the opening cohesive strength is a material constant value, while the critical opening displacement is a function of the crack opening rate.

Therefore the linear cohesive law is enriched with the following relationship for the rate-behavior of the critical opening:

$\delta_{c}=\delta_{c o}\left(1+\frac{\dot{\delta}}{\dot{\delta}_{o}}\right)$

where $\delta_{c o}$ corresponds to the critical crack opening displacement for the rate-independent law (static value) and $\delta_{0}$ represents a scaling crack opening rate. The introduction of this rate-dependent crack opening increases the value at which complete separation of the cohesive zone occurs with increasing opening rate. This should also lead to a higher dissipated fracture energy, which could be related

Table 3

Interface length between aggregates and matrix for the three different specimens.

\begin{tabular}{llll}
\hline Aggregate's number & 66 & 297 & 1198 \\
\hline Interface length $[\mathrm{m}]$ & 1.69 & 3.58 & 7.17 \\
\hline
\end{tabular}

with a more diffuse micro-cracking that the actual meso-scale is not able to capture.

We ran several simulations with this modified cohesive law for different loading rates in order to verify the strain rate-dependence. Surprisingly, we could not notice a remarkable difference in the peak strength values and dissipated fracture energy with the rateindependent law. This may be related with the chosen sample's size, for which one we are in presence of a mixed strength and toughness controlled regime.

Therefore, we decided to use a second variant where both $\sigma_{\mathrm{c}}$ and $\delta_{\mathrm{c}}$ are sensitive to the loading rate. This results in a law, which expands outwards with increasing loading rate as shown in Fig. 20a). The inconvenient with this kind of law is that the cohesive strength can be several times higher than the macroscopic material strength, which is physically questionable. Moreover, because we are assuming an initial rigid behavior (explicit approach), the cohesive strength needs to be known a priori. Therefore, we decided to link the law directly with the macroscopic loading ratio, introducing the following strain rate dependencies:

$f_{c t}=f_{c o}\left(1+\frac{\dot{\varepsilon}}{\dot{\varepsilon}_{0}}\right)$

$\delta_{c}=\delta_{c o}\left(1+\frac{\dot{\varepsilon}}{\dot{\varepsilon}_{o}}\right)$

where $\delta_{\text {co }}$ and $f_{\text {co }}$ correspond to the static values of cohesive strength and critical opening displacement respectively. Thus the two values are increased of the same amount with increasing loading rate (Fig. 20b)).

Thanks to the simple loading condition and geometry of the presented specimens, this second variant has been implemented specifically for the proposed virtual experimental setup. For more complicated loading conditions, it would be necessary to bound the strain-rate dependence with the deformation rate of the four gauss points adjacent to the inter-element boundary. Another possibility would be to take the static critical stress for insertion and to add, within the cohesive law, a rate-dependent viscosity parameter which depends on the opening rate (for instance [18]). A different approach, which would not involve a cohesive-approach, would consist in having a visco-plastic bulk material (such as proposed in combination with a continuum damage formulation in [13]).

As for the previous variant, we ran several simulations varying the loading rate. The resulting peak strengths are depicted in the graph of 
a

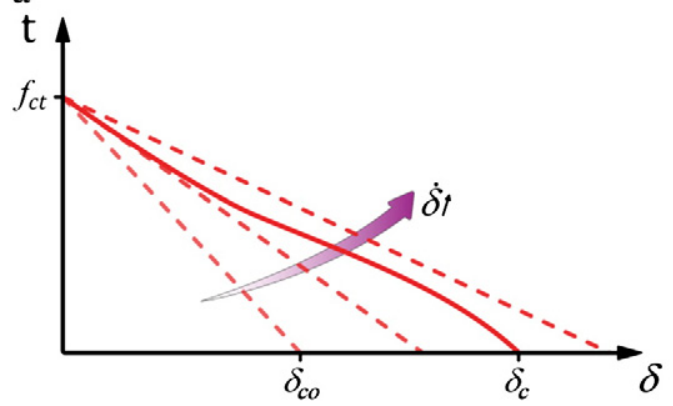

b

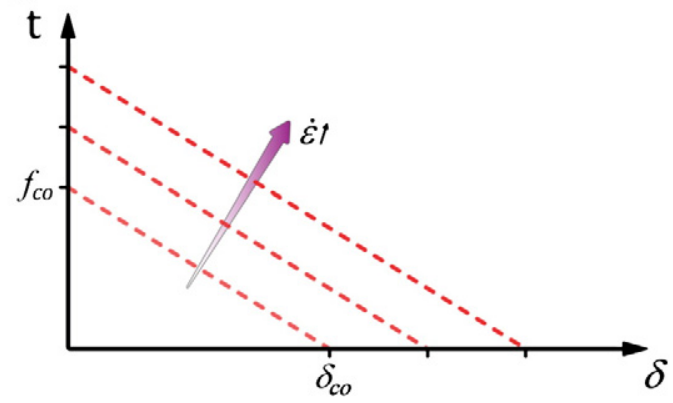

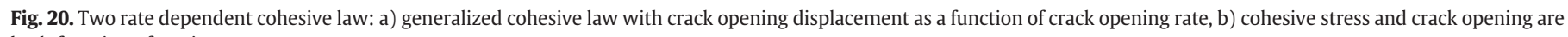
both function of strain rate.

Fig. 21a). One can remark that with this law the increase in peak strength and dissipated fracture energy is much more pronounced and is therefore closer to the experimental values (Fig. 21b)) and a better agreement can be simply reached by calibrating with the experimental values.

The fit could be perfected by choosing a non-linear relationship for $\sigma_{\mathrm{c}}$ and $\delta_{\mathrm{c}}$ (Eqs. (15a) and (15b)).

\section{Conclusions}

In this paper we have developed a 2D cohesive zone mesomechanical and dynamic model of concrete to study its fracturing behavior under low and high strain rate loadings. Although it is hard to operate in a strict toughness controlled regime, the meso-scale model has the significant advantage to represent aggregates and mortar explicitly. Both continuum phases are considered to behave elastically while initiation, coalescence and propagation of cracks are explicitly modeled by an automatic insertion of interface elements. We have investigated the influence of the aggregate's properties (e.g. referred to soft and hard aggregates, which differ in toughness and strength, but have a narrow range of elastic stiffness), the effect of the internal meso-structure (arrangement and shape of the aggregates) and the influence of their sizes on the simulations. From the obtained results we can extract the following salient conclusions.

While the constitutive law used to model the interfacial behavior is insensitive to the loading rate, the model is able to capture the increase in peak strength and strain at failure with increasing rate of loading. The increase in strength with the strain rate resides in a more diffuse micro-cracking and is related to the intrinsic characteristic opening time of the cohesive law. However, the dissipated fracture energy at peak strength seems to be roughly independent of strain rate (although, post-peak dissipation of energy is clearly an increasing function of strain rate). Therefore, for our meso-mechanical approach, we cannot discard the combination of inertial with material rate hardening mechanisms. The introduction of a rate dependent cohesive law increases the difference of the computed peak strength between low and high strain rates, leading to a closer match with experiments.

In addition, simulations carried out for two different types of aggregates (soft and hard) concluded that the peak strength is considerably sensitive to the toughness of the inclusions, but only at low strain rates. There, the crack patterns for the two different aggregates are different. However, at high strain rates, micro-cracks nucleate in the matrix and have no time to propagate in the aggregates before peak strength is reached. This lead to a similar stress-strain behavior for the two aggregate types (inertial forces dominate the overall behavior in terms of strength).

The material parameters used in this study have been taken from the recent experimental literature [32,31] without modification. For instance one should notice that the stiffness of both types of inclusions is lower than the one of the matrix paste. Therefore, in future work, we plan to extend the range of stiffness (and strength) of the constituents in order to calibrate our model with other experimental data and to establish the domain of validity of the above observations. Note that increasing the aggregates stiffness (and decreasing the interface strength) may also lead to a reduction of the (slight) meshdependency that the model exhibits at very high strain rates.
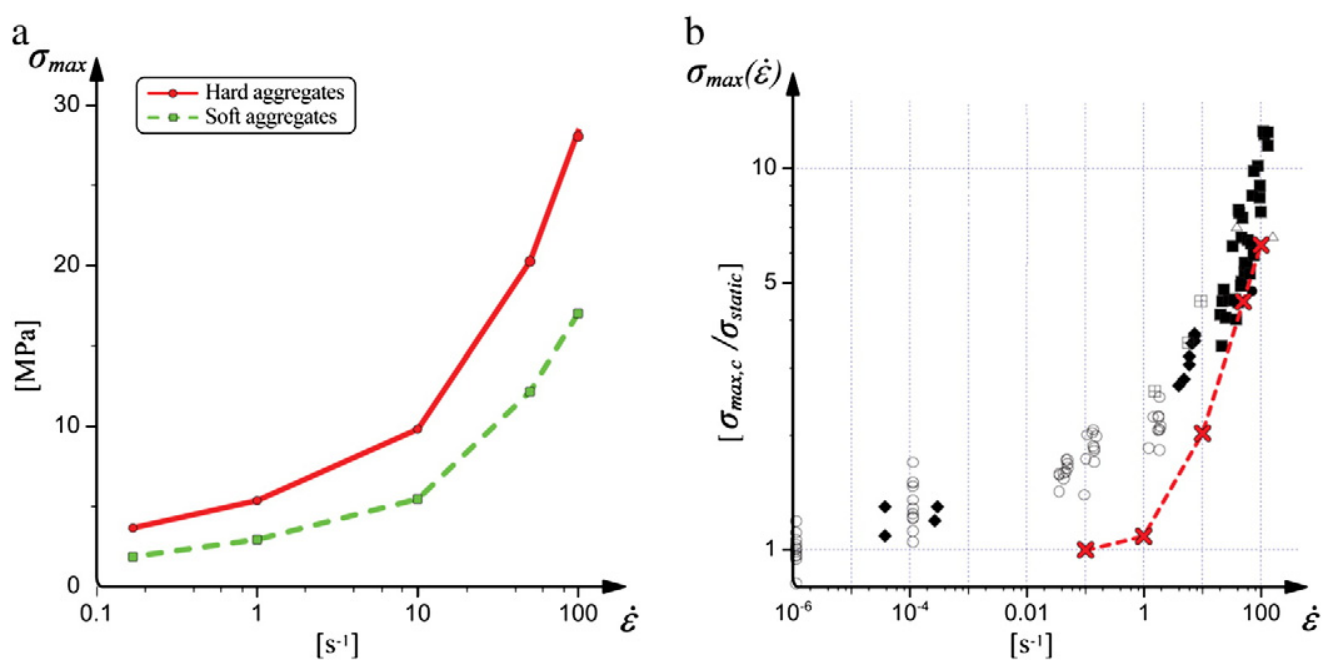

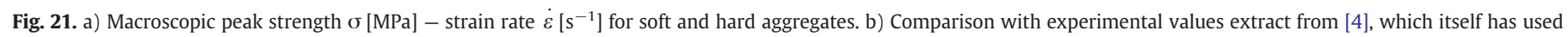
following experimental data $[5,30,1,38,21,15]$. 
A comparison between three different meso-structures considered brings the following remarks. The specimen with uniform mesostructure reduces the maximum strength, because of the aligned aggregate's faces with the loading direction. Since this is avoided by rotating the aggregates, the rotated meso-structure shows always the higher peak strength. Finally, the random distribution increases the probability to have weak zones (interfaces between aggregates and matrix are closer), which reduces the peak strength.

Finally, our results show that, having two concrete materials with same aggregate volume ratio, the one with smaller inclusions will exhibit a larger strength at low strain rate in case of soft aggregates. Large aggregates facilitate the propagation of cracks within inclusions. This involves a larger area of broken aggregates, which is linked with a lower strength.

With this work we presented a study of Mode-I fracture in concrete with a meso-structure. Our aim is to expand the model towards an engineering scale. We are now incorporating a contact algorithm in the code (which is an important improvement of the model in order to deal with compressive cracking) and our goal would be to be able to simulate failure of a simple reinforced concrete structure (like for instance a punching beam), where aggregates are supposed to play an important role, once meso-cracks are opened and in frictional contact.

\section{Acknowledgments}

This material is based on the work supported by the Swiss National Foundation under Grant no. 200021 122046/1.

\section{References}

[1] Birkimer, D.L., (1968). Critical normal fracture strain of cement portland concrete, Ph.D. thesis, University of Cincinnati, Ohio.

[2] J.E. Bolander, S. Saito, Fracture analysis using spring networks with random geometry, Engineering Fracture Mechanics 61 (1998) 569-591.

[3] A. Brara, J.R. Klepaczko, Fracture energy of concrete at high loading rates in tension, International Journal of Impact Engineering 34 (2007) 424-435.

[4] A. Brara, J.R. Klepaczko, Experimental characterization of concrete in dynamic tension, Mechanics of Materials 38 (2006) 253-267.

[5] Brara, A., (1999). Experimental study of dynamic tension of concrete via spalling, Ph.D. thesis, Laboratory of Physics and Mechanics of Materials, Metz University, France (in French).

[6] I. Carol, C. Lopez, O. Roa, Micromechanical analysis of quasi-brittlematerials using fracture based interface elements, International Journal for Numerical Methods in Engineering 52 (2001) 193-215.

[7] A. Caballero, I. Carol, C.M. Lopez, 3D meso-structural analysis of concrete specimens under uniaxial tension, Computer Methods in Applied Mechanics and Engineering 195 (52) (2006) 7182-7195.

[8] G.T. Camacho, M. Ortiz, Computational modeling of impact damage in brittle materials, Interantional Journal of Solids and Structures 33 (20-22) (1996) 2899-2938.

[9] J.F. Dube, G. Pijaudier-Cabot, C. La Borderie, A rate dependent damage model for concrete in dynamics, Journal of Engineering Mechanics ASCE 122 (1996) 939-947.

[10] F. Gatuingt, G. Pijaudier-Cabot, Coupled damage and plasticity modelling in transient dynamic analysis of concrete, International Journal for Numerical and Analytical Methods in Geomechanics 26 (2002) 1-24.

[11] G.V. Guinea, K. El-Sayed, C.G. Rocco, M. Elices, J. Planas, The effect of the bond between the matrix and the aggregates on the cracking mechanism and fracture parameters of concrete, Cement and Concrete Research 32 (2002) 1961-1970.

[12] P. Grassl, M. Jirásek, Meso-scale approach to modelling the fracture process zone of concrete subjected to uniaxial tension, International Journal of Solids and Structures 47 (2010) 957-968.

[13] G. Hervé, F. Gatuingt, A. Ibrahimbegovic, On numerical implementation of a coupled rate dependant damage-plasticity constitutive model for concrete in application to high-rate dynamic, Engineering Computations 22 (5-6) (2005) 583-604.

[14] R.H. Kraft, J.F. Molinari, K.T. Ramesha, D.H. Warner, Computational micromechanics of dynamic compressive loading of a brittle polycrystalline material using a distribution of grain boundary properties, Journal of the Mechanics and Physics of Solids 56 (8) (2008) 2618-2641.

[15] R. John, T. Antoun, A.M. Rajendran, Effect of strain rate and size on tensile strength of concrete, Proceedings, 1991 APS Topical Conference on Shock Compression of Condensed Matter, Elsevier Sci. Publ, Williamsburg, VA, 1992, pp. 501-504.

[16] J.W. Ju, On energy-based coupled elastoplastic damage theories: constitutive modeling and computational aspects, International Journal of Solids and Structures 25 (7) (1989) 803-833.
[17] J. Kozicki, J. Tejchman, Effect of aggregate structure on fracture process in concrete using 2D lattice model, 35th Solid Mechanics Conference Cracow, Poland, 59, 2007, pp. 365-384.

[18] D.V. Kubair, P. Geubelle, Y.Y. Huang, Analysis of a rate-dependent cohesive mode for dynamic crack propagation, Engineering Fracture Mechanics 70 (2003) 685-704.

[19] F. Lagier, X. Jourdain, C. De Sa, F. Benboudjema, J.B. Colliat, Numerical strategies for prediction of drying cracks in heterogeneous materials: comparison upon experimental results, Engineering Structures 33 (2011) 920-931.

[20] D.E. Lambert, C.A. Ross, Strain rate effects on dynamic fracture and strength, International Journal of Impact Engineering 24 (2000) 985-998.

[21] M.K. Mc Vay, Spall damage of concrete structures, Technical Report SL-88-22, US Army Corps of Engineers, Waterways Experiment Station, Vicksburg, Miss., USA, 1988.

[22] L.J. Malvar, C.A. Ross, Review of strain rate effects for concrete in tension, ACl Materials Journal 95 (6) (1998) 735-739.

[23] O. Miller, L.B. Freund, A. Needleman, Modeling and simulation of dynamic fragmentation in brittle materials, International Journal of Fracture 96 (2) (1999) 101-125.

[24] T. Nguyen, C. Lawrence, C. La Borderie, G. Nahas, A mesoscopic model for a better understanding of the transition from diffuse damage to localized damage, European Journal of Environmental and Civil Engineering 14 (6-7) (2010) 751-776.

[25] A.C. Palmer, J.R. Rice, The growth of slip surfaces in the progressive failure of overconsolidated clay, Proceedings of the Royal Society of London, Series A 332 (1973) 527-548.

[26] R.R. Pedersen, A. Simone, M. Stroeven, L.J. Sluys, Mesoscopic modelling of concrete under impact, Fracture Mechanics of Concrete and Concrete Structures 1-3 (2007) 571-578

[27] A. Pandolfi, M. Ortiz, Finite-deformation irreversible cohesive elements for three dimensional crack-propagation analysis, International Journal for Numerical Methods in Engineering 74 (1999) 1393-1420.

[28] J.R. Rice, The mechanics of earthquake rupture, in: A.M. Dziewonski, E. Boschi (Eds.), In Physics of the Earth's Interior (Proceedings of International School of Physics 'Enrico Fermi'), Amsterdam, North-Holland, 1980, pp. 555-649.

[29] P. Roelfstra, H. Sadouki, F. Wittmann, Le béton numerique, Materials \& Structures RILEM 18 (1985) 309-317.

[30] C.A. Ross, J.W. Tedesco, S.T. Kuennen, Effects of strain rate on concrete strength, ACI Materials Journal 92 (1995) 37-47.

[31] C. Rossello, M. Elices, Fracture of model concrete: 1. Types of fracture and crack path, Cement and Concrete Research 34 (2004) 1441-1450.

[32] C. Rossello, M. Elices, G.V. Guinea, Fracture of model concrete: 2. Fracture energy and characteristic length, Cement and Concrete Research 36 (2006) 1345-1353.

[33] P. Rossi, J.G.M. van Mier, F. Toutlemonde, F. Maou, C. Boulay, Effect of loading rate on the strength of concrete subjected to uniaxial tension, Materials and Structures 27 (1994) 260-264.

[34] G. Ruiz, A. Pandolfi, M. Ortiz, Three-dimensional cohesive modeling of dynamic mixed-mode fracture, International Journal for Numerical Methods in Engineering 52 (2001) 97-120.

[35] E. Schlangen, J.G.M. van Mier, Simple lattice model for numerical simulation of fracture of concrete materials and structures, Materials and Structures 25 (1992) 534-542.

[36] T. Stankowski, K. Runesson, S. Sture, et al., Simulation of progressive failure in particle composites, Micromechanics of Failure of Quasi-Brittle Materials, 1990, pp. 285-294.

[37] L.J. Sluys, R. de Borst, Wave propagation and localization in a rate-dependent cracked medium model formulation and one dimensional examples, Interantional Journal of Solids and Structures 29 (1992) 2945-2958.

[38] Toutlemonde, F., (1994). Shock strength of concrete structures; From material behaviour to structure design, Doctoral Thesis, E.N.P.C., Paris (in French).

[39] M.G.A. Tijssens, L.J. Sluys, E. van der Giessen, Simulation of fracture of cementitious composites with explicit modeling of microstructural features, Engineering Fracture Mechanics 68 (11) (2001) 1245-1263.

[40] J.C.A.M. Van Doormaal, J. Weerheijm, L.G. Sluys, Experimental and numerical determination of the dynamic fracture energy of concrete, Journal of Physics IV 4 (1994) 501-506.

[41] R.A. Vonk, H.S. Rutten, J.G.M. Van Mier, Micromechanical simulation of concrete softening, Fracture Processes in Concrete, Rock and Ceramics, Vols 1 and 2, 13, 1991, pp. 129-138.

[42] F. Wittmann, Structure of concrete with respect to crack formation, in: F. Wittmann (Ed.), Fracture Mechanics of Concrete, Elsevier Science Publishers, 1983, pp. 43-74

[43] D. Yan, G. Lin, Dynamic properties of concrete in direct tension, Cement and Concrete Research 36 (2006) 1371-1378.

[44] F. Zhou, J.F. Molinari, T. Shioya, A rate-dependent cohesive model for simulating dynamic crack propagation in brittle materials, Engineering Fracture Mechanics 72 (2005) 1383-1410.

[45] G. Cusatis, D. Pelessone, Mesolevel simulation of reinforced concrete structures under impact loadings, Computational Modelling of Concrete Structures, EURO-C Conference (2006) 63-70.

[46] D.S. Dugdale, Yielding of steel sheets containing clits, Journal of the Mechanics and Physics of Solids 8 (1960) 100-104.

[47] G.I. Barenblatt, The Mathematical Theory of Equilibrium Cracks in Brittle Fracture Advances in Applied Mechanics 7 (1962) 55-129.

[48] A. Caballero, 3D meso-mechanical numerical analysis of concrete using interface elements. PhD thesis (2005), ETSECCPB-UPC, E-08034 Barcelona (Spain). 